\title{
Live birth achieved despite the absence of ejaculated spermatozoa and mature oocytes retrieved: a case report
}

\author{
Zuzana Holubcová $^{1,2}$ (D) $\cdot$ Pavel Otevřel $^{1} \cdot$ Marek Koudelka $^{1} \cdot$ Soňa Kloudová ${ }^{1}$
}

Received: 27 October 2020 / Accepted: 10 January 2021 / Published online: 20 January 2021

(C) The Author(s) 2021

\begin{abstract}
The most common reason for in vitro fertilization (IVF) cycle cancelation is a lack of quality gametes available for intracytoplasmic sperm injection (ICSI). Here we present the successful fertility treatment of the couple affected by obstructive azoospermia combined with suboptimal response to controlled ovarian stimulation. Since the conventional approach appeared ineffective to overcome both partners' specific problems, the targeted interventions, namely, (1) pharmacological enhancement of sperm motility and (2) polarized light microscopy (PLM)-guided optimization of ICSI time, were applied to rescue the cycle with only immature oocytes and immotile testicular sperm retrieved. The treatment with theophylline aided the selection of viable spermatozoa derived from cryopreserved testicular tissue. When the traditional stimulation protocol failed to produce mature eggs, non-invasive spindle imaging was employed to adjust the sperm injection time to the maturational stage of oocytes extruding a polar body in vitro. The fertilization of 12 late-maturing oocytes yielded 5 zygotes, which all developed into blastocysts. One embryo was transferred into the uterus on day 5 post-fertilization, and another 3 good quality blastocysts were vitrified for later use. The pregnancy resulted in a full-term delivery of a healthy child. This case demonstrates that the individualization beyond the standard IVF protocols should be considered to maximize the chance of poor-prognosis patients to achieve pregnancy with their own gametes.
\end{abstract}

Keywords IVF add-ons $\cdot$ Oocyte maturity $\cdot$ Polarized light microscopy $\cdot$ Theophylline $\cdot$ Testicular sperm

\section{Introduction}

Assisted reproductive techniques (ART) evolved from simple co-incubation of gametes into a broad repertoire of procedures helping to deal with various fertility issues and avoid transmission of genetic diseases. The concept of controlled ovarian stimulation was developed to provide multiple eggs for insemination by ejaculated sperm. However, patients show variability in their response to hormonal treatment $[1,2]$. Occasionally, oocytecumulus complexes recovered from preovulatory follicles contain only immature oocytes instead of mature eggs. In the

Zuzana Holubcová

zholub@med.muni.cz

1 Reprofit International, Clinic of Reproductive Medicine, Hlinky 122, 60300 Brno, Czech Republic

2 Department of Histology and Embryology, Faculty of Medicine, Masaryk University, Masaryk University Campus - building A1, Kamenice 3, 62500 Brno, Czech Republic absence of metaphase II (MII) oocytes, the cycle is typically canceled, and immature oocytes are discarded. Failure to complete maturation in the expected timeframe might reflect intrinsic disturbances that compromise female gamete's developmental potential [3-6]. Nevertheless, some metaphase I (MI) oocytes which extruded a polar body $(\mathrm{PB})$ in vitro were shown to be clinically utilizable and give rise to pregnancies [7-10]. Ensuring that developmentally delayed oocytes reached the MII stage before ICSI prevents untimely sperm injection and enhances the chance of successful fertilization [11-14]. Polarized light microscopy (PLM) serves as a tool to inspect the presence of MII spindle and time ICSI in accordance with the oocyte's maturational stage [15]. Late-maturing oocytes capable of assembling a PLM-detectable MII spindle increase the pool of injectable eggs, thus providing hope for women whose response to gonadotropins is inadequate.

Similarly, testicular sperm extraction (TESE) constitutes the last recourse for men who have no sperm in their ejaculate $[16,17]$. However, immature spermatozoa retrieved from extracted testicular tissue are often pathological and immotile. Compounds interfering with phosphodiesterase (PDE) 
activity, such as pentoxifylline, are known to raise intracellular cyclic adenosine monophosphate (cAMP) and boost motility in asthenospermic samples. Similarly, incubation of TESE-derived sperm with another PDE inhibitor, dimethylxanthine theophylline, enhances sperm movement, thus allowing embryologists to distinguish between viable, yet immotile, and non-viable male gametes [17-19]. So far, no negative effects of sperm stimulation were reported on ICSI outcomes [20-27]. Nevertheless, the potential risks related to the drug exposition still raise safety concerns.

This report provides detailed information on how the application of optional ART techniques, namely, (1) pharmacological stimulation of motility in frozen-thawed testicular sperm and (2) PLM-guided optimization of ICSI time for oocytes extruding PB in vitro, helped to achieve a successful pregnancy under extremely challenging circumstances.

\section{Results}

\section{Patients' characteristics and previous treatment}

In 2016, a couple with a history of 3 failed attempts performed at a different IVF center approached the clinic presenting with primary male infertility due to the congenital bilateral absence of vas deferens and risk of ovarian hyperstimulation syndrome (OHSS). The previous genetic testing revealed that the 31year-old male patient was a CTFR gene carrier while his 32year-old partner was a healthy homozygote. He was referred for TESE, and spermatozoa isolated from the freshly retrieved testicular biopsy were used for ICSI. The rest of the testicular sperm sample was cryopreserved. The nulligravid non-obese female with oligomenorrhea was diagnosed with polycystic ovaries based on ultrasound examination and hormonal profile. To ameliorate the risk of OHSS, she received metformin pre-treatment. Subsequently, the patient underwent ovarian stimulation using antagonist protocol and recombinant follicle-stimulating hormone (rFSH) (Gonal-f 112 IU, Merck Serono, Switzerland). Human chorionic gonadotropin (hCG) (Pregnyl 5.000 IU, Organon, Netherlands) was used to induce ovulation. The oocyte pickup was scheduled for $35 \mathrm{~h}$ after the triggering injection. The cumulus-oocyte complexes were retrieved using ultrasound-guided transvaginal aspiration and collected in dual buffered MHM medium (\#90166, Irvine Scientific, USA). The oocyte denudation was carried out $10 \mathrm{~min}$ after retrieval to distinguish the in vivo matured eggs from oocytes that complete maturation in vitro and benefit from prolonged incubation before ICSI [14, 28]. Despite a good follicular count, no PB-displaying oocytes were obtained. To allow completion of the maturational program, retrieved MI oocytes were placed into CSCM-C medium (\#90165, Irvine Scientific, USA) covered with mineral oil and incubated at $37{ }^{\circ} \mathrm{C}$ with a humidified atmosphere of $5 \%$
$\mathrm{O}_{2}$ and $6 \% \mathrm{CO}_{2}$. Four hours later, 2 out of 7 immature oocytes exhibited a PB and were injected with TESE-derived spermatozoa. Both oocytes showed two well-defined pronuclei 17$18 \mathrm{~h}$ after ICSI, but no blastocyst was obtained.

\section{Case report}

In 2018, the couple returned to the clinic to start another cycle. This time, the stimulation phase was prolonged to 13 days of rFSH application (Puregon 125 IU, MSD, USA) to promote follicular growth. The trigger shot was scheduled for 2 days after the biggest follicles reached a diameter of $18 \mathrm{~mm}$, and oocyte retrieval was undertaken $36 \mathrm{~h}$ after the $\mathrm{hCG}$ injection. Nevertheless, no mature eggs but 17 immature oocytes were retrieved. Only 1 out of 17 MI oocytes displayed a PB after $3 \mathrm{~h}$ of in vitro incubation, at standard ICSI time. However, this oocyte did not show an MII spindle signal during the PLM examination, indicating that the maturation process was not yet completed. Therefore, ICSI was postponed, and the maturation stage of each oocyte was closely monitored, as previously described [28]. During the next $2 \mathrm{~h}$, another 4 oocytes extruded PBs. However, they all lacked a PLM-detectable MII spindle signal. Sperm injection was undertaken $2 \mathrm{~h}$ later $(43 \mathrm{~h}$ after hCG) when a total of 12 oocytes exhibited PBs, and 10 of them showed MII spindles. The maturational timelines of individual oocytes, together with their fertilization outcomes, are summarized in Table 1.

The sample derived from the male patient's testicular biopsy was thawed, but all recovered spermatozoa exhibited pathological morphology and imperceptible spontaneous motility. To prevent ICSI with non-viable spermatozoa, we pretreated the sample with the theophylline-containing medium (SpermMobil, GM501, Gynemed, Germany) for $10 \mathrm{~min}$ according to the manufacturer's instructions. Short-term exposure to theophylline immediately promoted residual sperm motility and decreased the time necessary to find the best available male gametes for the injection. Each spermatozoon selected for fertilization was extensively rinsed at least three times in the MHM medium before ICSI. Sperm-injected oocytes were transferred to individual droplets of CSCM-C medium, and their post-fertilization fates were tracked.

The next day, 5 out of 12 sperm-injected oocytes showed signs of fertilization. These 5 embryos (all originating from spindled oocytes) reached the blastocyst stage after extended cultivation. Trophectoderm biopsy and genetic testing were proposed, but the couple made an informed decision to proceed with one fresh embryo transfer on day 5 and 3 good quality blastocysts cryopreserved. A singleton pregnancy culminated with spontaneous vaginal delivery at the 38 th week of gestation. The male newborn had a normal birthweight ( $3010 \mathrm{~g})$, and no congenital defects were reported. The child's well-being was confirmed at the age of 1 year by a detailed 
Table 1 Maturity status of individual oocytes and their postfertilization outcome

\begin{tabular}{llllll}
\hline Oocyte \# & $\begin{array}{l}\text { hCG }+38 \mathrm{~h} \\
\text { stage/ } \\
\text { spindle }\end{array}$ & $\begin{array}{l}\text { hCG }+43 \mathrm{~h} \\
\text { stage/ } \\
\text { spindle }\end{array}$ & Fertilization & Blastulation & Utilization \\
\hline 1 & MII/no & MII/yes & No & No & No \\
2 & MI/NA & MII/yes & No & No & No \\
3 & MI/NA & MII/yes & Yes & Yes & Yes \\
4 & MI/NA & MII/yes & Yes & Yes & No \\
5 & MI/NA & MII/yes & No & No & No \\
6 & MI/NA & MII/yes & Yes & Yes & Yes \\
7 & MI/NA & MII/yes & Yes & Yes & Yes \\
8 & MI/NA & MII/yes & No & No & No \\
9 & MI/NA & MII/yes & No & No & No \\
10 & MI/NA & MII/yes & Yes & Yes & Yes \\
11 & MI/NA & MII/no & No & No & No \\
12 & MI/NA & MII/no & No & No & No \\
\hline
\end{tabular}

survey and will be further monitored to evaluate the treatment 's safety.

\section{Discussion}

Over 40 years ago, human IVF emerged as a highly controversial experimental technique. Nowadays, fertility clinics are urged to refrain from advertising unproven methods [29]. The recently developed traffic light system disproved the use of add-on procedures until credible evidence on safety and effectivity is provided [30]. However, many ART techniques routinely used today have been introduced into clinical practice even though their benefits were not demonstrated in randomized controlled trials. Moreover, some fertility issues affect only a small population of IVF patients, and large clinical studies are difficult to design. Innovations are supposed to drive ART advancement, but rigid demand for evidencebased practice might hinder building supportive evidence for novel techniques and alternative approaches [31]. Sharing experience concerning a bona fide use of unconventional interventions and adjuvant therapies is essential in rare patient phenotypes. Clinicians are obliged to monitor the obstetrical and neonatal complications and report any adverse effects that may be recognized. Synthesis and assessment of a "critical mass" of clinical data is a vital prerequisite to evaluate the efficacy of empirical treatments that hold the potential to improve management of ART cycles with bad prognosis.

Here we describe a peculiar case in which the modification of conventional fertility treatment made a difference for the couple with little chance to conceive using traditional IVF protocols. The published data support the use of sperm motility enhancement with theophylline [23-27] and rescue in vitro maturation of late-maturing oocytes [11-14]. Nevertheless, until a sufficient body of evidence is accumulated, these methods must still be regarded as experimental. Our patients were thoroughly counseled over the character of the treatment, and written informed consent was obtained. The follow-up revealed no pregnancy complications or anomalies in the offspring. The health parameters of the child will be further monitored to assess the long-term consequences of the treatment. Based on this experience and previous reports, we suggest considering this approach to salvage cycles with a lack of gametes available for fertilization.

There are some critical aspects and limitations of the procedures in question which need to be acknowledged. Pharmacological stimulation enables faster and more accurate identification of viable sperm in pathological samples, thus increasing the chance for a successful outcome in IVF cycles affected by severe male infertility factor. Availability of ready-to-use and quality-controlled theophylline-containing commercial media makes the application of sperm motility enhancement possible in countries where self-preparing and in-house supplementation of IVF-used solutions are prohibited. Brief exposition to motility-inducing agents appears not to impair sperm genetic integrity. Nevertheless, extensive washing of selected spermatozoa is highly recommended as a precaution to prevent adverse effects on oocytes and embryos [17, 32]. Since spermatogenic impairment was found to increase the risk of embryonic mosaicism, preimplantation genetic testing of ICSI outcomes is advisable when TESE-derived sperm is used [33-35].

The principle and methodology of PLM examination, as well as the optimization of the ICSI timing, have been thoroughly described previously [14, 15, 28, 36]. However, it is important to emphasize here that spindle imaging provides no information about chromatin configuration at the metaphase plate. Chromosome misalignment and precocious chromatid splitting could occur in oocytes featuring the MII spindle [28, 37]. Therefore, younger patients with an unexpectedly 
inadequate response to ovarian stimulation due to specific genetic background and/or endocrine derangements [38-40] are more likely to benefit from PLM-navigated ICSI timing than women of advanced age.

In conclusion, our patient's story highlights the need for personalization and responsible innovation in reproductive medicine. While unproven therapies cannot be recommended in a general context, the judicious use of specific unconventional interventions is justifiable as an alternative to cycle cancelation.

Acknowledgments The authors wish to thank the embryology laboratory and the staff of Reprofit International for undertaking laboratory procedures, documentation, and follow-up of this patient case.

Authors' contribution Z.H.: data analysis and interpretation and manuscript writing; P.O.: data analysis (gynecology) and manuscript revision; M.K.: clinical management of the treatment cycle and manuscript revision; S.K.: data analysis (embryology and andrology) and manuscript revision. All authors substantially contributed to the study and approved the final manuscript.

Funding $\mathrm{R} \& \mathrm{D}$ funds of the clinic covered costs related to the application of additional IVF procedures.

\section{Compliance with ethical standards}

Conflict of interest The authors declare that they have no conflict of interest.

Ethics approval The clinic's ethical committee approved the use of supplementary IVF procedures where appropriate. Both partners signed informed consent with each non-standard procedure.

Open Access This article is licensed under a Creative Commons Attribution 4.0 International License, which permits use, sharing, adaptation, distribution and reproduction in any medium or format, as long as you give appropriate credit to the original author(s) and the source, provide a link to the Creative Commons licence, and indicate if changes were made. The images or other third party material in this article are included in the article's Creative Commons licence, unless indicated otherwise in a credit line to the material. If material is not included in the article's Creative Commons licence and your intended use is not permitted by statutory regulation or exceeds the permitted use, you will need to obtain permission directly from the copyright holder. To view a copy of this licence, visit http://creativecommons.org/licenses/by/4.0/.

\section{References}

1. Oudendijk JF, Yarde F, Eijkemans MJ, Broekmans FJ, Broer SL. The poor responder in IVF: is the prognosis always poor?: a systematic review. Hum Reprod Update. 2012;18:1-11.

2. Rustamov O, Wilkinson J, La Marca A, Fitzgerald C, Roberts SA. How much variation in oocyte yield after controlled ovarian stimulation can be explained? A multilevel modelling study. Hum Reprod Open. 2017;2017:hox018.

3. Combelles CM, Cekleniak NA, Racowsky C, Albertini DF. Assessment of nuclear and cytoplasmic maturation in in-vitro matured human oocytes. Hum Reprod. 2002;17:1006-16.
4. Coticchio G, Dal Canto M, Mignini Renzini M, Guglielmo MC, Brambillasca F, Turchi D, et al. Oocyte maturation: gamete-somatic cells interactions, meiotic resumption, cytoskeletal dynamics and cytoplasmic reorganization. Hum Reprod Update. 2015;21:42754.

5. Ko DS, Lee SH, Park DW, Yang KM, Lim CK. Pregnancy and fertilization potential of immature oocytes retrieved in intracytoplasmic sperm injection cycles. Clin Exp Reprod Med. 2015;42:118-25.

6. Vellez LT, Brogliato C, Berton CZ, Yoshida IH, Barbosa CP, Cordts EB. ICSI in late matured oocytes, is it worth it? Study with laboratory, clinical and genetic evaluation results. JBRA Assist Reprod. 2020;24:173-4.

7. Piqueras P, Gallardo M, Hebles M, Jiménez JM, Migueles B, Montero L, et al. Live birth after replacement of an embryo obtained from a spontaneously in vitro matured metaphase-I oocyte. Syst Biol Reprod Med. 2017;63:209-11.

8. Shu Y, Gebhardt J, Watt J, Lyon J, Dasig D, Behr B. Fertilization, embryo development, and clinical outcome of immature oocytes from stimulated intracytoplasmic sperm injection cycles. Fertil Steril. 2007;87:1022-7.

9. De Vos A, Van de Velde H, Joris H, Van Steirteghem A. In-vitro matured metaphase-I oocytes have a lower fertilization rate but similar embryo quality as mature metaphase-II oocytes after intracytoplasmic sperm injection. Hum Reprod. 1999;14:1859-63.

10. Sachdev NM, Grifo JA, Licciardi F. Delayed intracytoplasmic sperm injection (ICSI) with trophectoderm biopsy and preimplantation genetic screening (PGS) show increased aneuploidy rates but can lead to live births with single thawed euploid embryo transfer (STEET). J Assist Reprod Genet. 2016;33:1501-5.

11. Montag M, Schimming T, van der Ven H. Spindle imaging in human oocytes: the impact of the meiotic cell cycle. Reprod BioMed Online. 2006;12:442-6.

12. Hyun CS, Cha JH, Son WY, Yoon SH, Kim KA, Lim JH. Optimal ICSI timing after the first polar body extrusion in in vitro matured human oocytes. Hum Reprod. 2007;22:1991-5.

13. Yu Y, Yan J, Liu ZC, Yan LY, Li M, Zhou Q, et al. Optimal timing of oocyte maturation and its relationship with the spindle assembly and developmental competence of in vitro matured human oocytes. Fertil Steril. 2011;96:73-8.e1.

14. Holubcová Z, Kyjovská D, Martonová M, Páralová D, Klenková T, Otevřel $\mathrm{P}$, et al. Egg maturity assessment prior to ICSI prevents premature fertilization of late-maturing oocytes. J Assist Reprod Genet. 2019;36:445-52.

15. Montag M, Köster M, van der Ven K, van der Ven H. Gamete competence assessment by polarizing optics in assisted reproduction. Hum Reprod Update. 2011;17:654-66.

16. Schlegel PN. Nonobstructive azoospermia: a revolutionary surgical approach and results. Semin Reprod Med. 2009;27:165-70.

17. Verheyen G, Popovic-Todorovic B, Tournaye H. Processing and selection of surgically-retrieved sperm for ICSI: a review. Basic Clin Androl. 2017;27:6.

18. Nordhoff V. How to select immotile but viable spermatozoa on the day of intracytoplasmic sperm injection? An embryologist's view. Andrology. 2015;3:156-62.

19. Tardif S, Madamidola OA, Brown SG, Frame L, Lefièvre L, Wyatt PG, et al. Clinically relevant enhancement of human sperm motility using compounds with reported phosphodiesterase inhibitor activity. Hum Reprod. 2014;29:2123-35.

20. Kovacic B, Vlaisavljevic V, Reljic M. Clinical use of pentoxifylline for activation of immotile testicular sperm before ICSI in patients with azoospermia. J Androl. 2006;27:45-52.

21. Amer M, Metawae B, Hosny H, Raef A. Beneficial effect of adding pentoxifylline to processed semen samples on ICSI outcome in infertile males with mild and moderate asthenozoospermia: a 
randomized controlled prospective crossover study. Iran J Reprod Med. 2013;11:939-44.

22. Terriou P, Hans E, Cortvrindt R, Avon C, Charles O, Salzmann J, et al. Papaverine as a replacement for pentoxifylline to select thawed testicular or epididymal spermatozoa before ICSI. Gynecol Obstet Fertil. 2015;43:786-90.

23. Ebner T, Tews G, Mayer RB, Ziehr S, Arzt W, Costamoling W, et al. Pharmacological stimulation of sperm motility in frozen and thawed testicular sperm using the dimethylxanthine theophylline. Fertil Steril. 2011;96:1331-6.

24. Ebner T, Shebl O, Mayer RB, Moser M, Costamoling W, Oppelt P. Healthy live birth using theophylline in a case of retrograde ejaculation and absolute asthenozoospermia. Fertil Steril. 2014;101:3403.

25. Ebner T, Maurer M, Oppelt P, Mayer RB, Duba HC, Costamoling $\mathrm{W}$, et al. Healthy twin live-birth after ionophore treatment in a case of theophylline-resistant Kartagener syndrome. J Assist Reprod Genet. 2015;32:873-7.

26. Wöber M, Ebner T, Steiner SL, Strohmer H, Oppelt P, Plas E, et al. A new method to process testicular sperm: combining enzymatic digestion, accumulation of spermatozoa, and stimulation of motility. Arch Gynecol Obstet. 2015;291:689-94.

27. Sandi-Monroy NL, Musanovic S, Zhu D, Szabó Z, Vogl A, Reeka $\mathrm{N}$, et al. Use of dimethylxanthine theophylline (SpermMobil). Arch Gynecol Obstet. 2019;300:1435-43.

28. Holubcová Z, Kyjovská D, Martonová M, Páralová D, Klenková T, Kloudová S. Human egg maturity assessment and its clinical application. J Vis Exp. 2019;150:e60058. https://doi.org/10.3791/ 60058.

29. Harper J, Jackson E, Sermon K, Aitken RJ, Harbottle S, Mocanu E, et al. Adjuncts in the IVF laboratory: where is the evidence for 'addon' interventions? Hum Reprod. 2017;32:485-91.

30. HFEA. Treatment add ons. [online] 2020 [ref. 2020-12-15] available on https:/www.hfea.gov.uk/treatments/explore-all-treatments/ treatment-add-ons/. Accessed 15 Dec 2020
31. Macklon NS, Ahuja KK, Fauser B. Building an evidence base for IVF 'add-ons'. Reprod BioMed Online. 2019;38:853-6.

32. Ortega C, Verheyen G, Raick D, Camus M, Devroey P, Tournaye H. Absolute asthenozoospermia and ICSI: what are the options? Hum Reprod Update. 2011;17:684-92.

33. Silber S, Escudero T, Lenahan K, Abdelhadi I, Kilani Z, Munné S. Chromosomal abnormalities in embryos derived from testicular sperm extraction. Fertil Steril. 2003;79:30-8.

34. Tarozzi N, Nadalini M, Lagalla C, Coticchio G, Zacà C, Borini A. Male factor infertility impacts the rate of mosaic blastocysts in cycles of preimplantation genetic testing for aneuploidy. J Assist Reprod Genet. 2019;36:2047-55.

35. Kahraman S, Sahin Y, Yelke H, Kumtepe Y, Tufekci MA, Yapan $\mathrm{CC}$, et al. High rates of aneuploidy, mosaicism and abnormal morphokinetic development in cases with low sperm concentration. J Assist Reprod Genet. 2020;37:629-40.

36. Caamaño JN, Muñoz M, Diez C, Gómez E. Polarized light microscopy in mammalian oocytes. Reprod Domest Anim. 2010;45(Suppl 2):49-56.

37. Wang WH, Keefe DL. Prediction of chromosome misalignment among in vitro matured human oocytes by spindle imaging with the PolScope. Fertil Steril. 2002;78:1077-81.

38. Casarini L, Santi D, Marino M. Impact of gene polymorphisms of gonadotropins and their receptors on human reproductive success. Reproduction. 2015;150:R175-84.

39. Diamanti-Kandarakis E. Polycystic ovarian syndrome: pathophysiology, molecular aspects and clinical implications. Expert Rev Mol Med. 2008;10:e3.

40. Johansson HKL, Damdimopoulou P, van Duursen MBM, Boberg J, Franssen D, de Cock M, et al. Putative adverse outcome pathways for female reproductive disorders to improve testing and regulation of chemicals. Arch Toxicol. 2020;94:3359-79.

Publisher's note Springer Nature remains neutral with regard to jurisdictional claims in published maps and institutional affiliations. 